\title{
On the use of control surface excitation in flutter testing
}

\section{J R Wright*, J Wong, J E Cooper and G Dimitriadis}

Dynamics and Aeroelasticity Research Group, Manchester School of Engineering, University of Manchester, UK

\begin{abstract}
Flutter testing is aimed at demonstrating that the aircraft flight envelope is flutter free. Response measurements from deliberate excitation of the structure are used to identify and track frequency and damping values against velocity. In this paper, the common approach of using a flight control surface to provide the excitation is examined using a mathematical model of a wing and control surface whose rotation is restrained by a simple actuator. In particular, it is shown that it is essential to use the demand signal to the actuator as a reference signal for data processing. Use of the actuator force (or strain) or control angle (or actuator displacement) as a reference signal is bad practice because these signals contain response information. It may also be dangerous in that the onset of flutter may not be seen in the test results.
\end{abstract}

Keywords: flutter testing, aircraft flight envelope, excitation, flight control

$\begin{array}{ll}\text { NOTATION } & \\ a & \text { two-dimensional lift curve slope } \\ a_{\mathrm{c}} & \text { lift per control angle } \\ a_{\mathrm{m}} & \text { pitching moment/control angle } \\ \mathbf{A} & \text { inertia matrix } \\ A_{\mathrm{F}} & \text { area of pressure stabilization piston } \\ A_{\mathrm{P}} & \text { piston area } \\ b_{1} & \text { hinge moment/incidence } \\ b_{2} & \text { hinge moment/control angle } \\ \mathbf{B} & \text { aerodynamic damping matrix } \\ B(s) & \text { Laplace transform of control rotation } \\ c & \text { chord/subscript for control surface } \\ \mathbf{C} & \text { aerodynamic stiffness matrix } \\ \mathrm{d} & \text { differential operator } \\ d_{1,2,3} & \text { actuator parameters } \\ \mathbf{D} & \text { structural damping matrix } \\ e & \text { normalized position of flexural axis } \\ \mathbf{E} & \text { structural stiffness matrix } \\ F & \text { force } \\ F_{\mathrm{P}} & \text { force applied to actuator } \\ h & \text { actuator/control lever arm } \\ H & \text { hinge moment } \\ H(s) & \text { transfer function } \\ I & \text { moment of inertia }\end{array}$

The MS was received on 5 August 2003 and was accepted after revision for publication on 5 November 2003.

* Corresponding author: Dynamics and Aeroelasticity Research Group, Manchester School of Engineering, University of Manchester, Oxford Road, Manchester M13 9PL, UK.

$\begin{array}{ll}i, j & \text { integers } \\ J & \text { actuator damping } \\ k & \text { stiffness } \\ K & \text { actuator stiffness } \\ K_{\mathrm{F}} & \text { stiffness of the stabilization spring } \\ K_{\mathrm{V}} & \text { valve flow constant } \\ K_{0} & \text { static stiffness } \\ K_{\infty} & \text { oil bounce stiffness } \\ L & \text { lift } \\ m & \text { mass/subscript for the main surface } \\ M & \text { pitching moment } \\ M_{\dot{\beta}} & \text { control damping derivative } \\ M_{\dot{\theta}} & \text { torsional damping derivative } \\ N & \text { bulk modulus of oil } \\ P(s) & \text { polynomial of system poles } \\ P_{\mathrm{J}} & \text { pressure difference across the piston } \\ P_{\mathrm{S}}, P_{\mathrm{R}} & \text { supply and return pressure } \\ P_{1}, P_{2} & \text { pressures in chambers } 1 \text { and } 2 \\ q & \text { generalized coordinate } \\ Q & \text { generalized force } \\ Q_{1}, Q_{2} & \text { valve flows into chambers } 1 \text { and } 2 \\ \mathbf{R} & \text { matrix } \\ s & \text { span/Laplace transform operator } \\ \mathbf{S} & \text { matrix } \\ t & \text { time } \\ \mathbf{T} & \text { kinetic energy/matrix } \\ T_{\mathrm{D}}(s) & \text { displacement transmissibility } \\ T_{\mathrm{F}}(s) & \text { force transmissibility } \\ T_{\beta} & \text { torque provided by actuator } \\ U & \text { potential energy } \\ \mathbf{U} & \text { matrix } \\ & \end{array}$

Proc. Instn Mech. Engrs Vol. 217 Part G: J. Aerospace Engineering 


$\begin{array}{ll}V & \text { velocity } \\ V_{\mathrm{o}} & \text { oil volume } \\ \mathbf{W} & \text { work done/matrix } \\ x & \text { chordwise coordinate } \\ x_{\mathrm{f}} & \text { distance to flexural axis } \\ x_{\mathrm{h}} & \text { distance to control hinge line } \\ X_{i} & \text { demanded displacement of actuator } \\ X_{\mathrm{V}} & \text { valve displacement } \\ X_{0} & \text { actuator body displacement } \\ y & \text { spanwise coordinate } \\ \mathbf{Y} & \text { matrix } \\ Y(s) & \text { Laplace transform of response } \\ Z(s) & \text { polynomial of system zeros } \\ Z_{\mathrm{A}}(s) & \text { actuator impedance } \\ \beta & \text { control rotation angle } \\ \beta_{i} & \text { effective demanded control angle } \\ \gamma & \text { angle of flap } \\ \delta & \text { increment } \\ \theta & \text { angle of twist } \\ \lambda & \text { eigenvalue } \\ \mu & \text { real part of the eigenvalue } \\ \mu & \text { mechanical gearing of the valve } \\ v & \text { frequency parameter } \\ \rho & \text { air density } \\ \omega & \text { frequency (rad/s) } \\ \omega \omega_{\mathrm{D}}, \omega_{\mathrm{F}} & \text { displacement and force cut-off frequency } \\ \text { overdot } & \text { d/d } t \\ \text { underscore } & \text { vector } \\ {[]} & \text { matrix } \\ & \end{array}$

\section{INTRODUCTION}

Aircraft flutter is an instability that involves two or more modes of vibration coupling together via aerodynamic forces such that energy is extracted from the airstream [1]. Once the flutter velocity is reached, the amplitude can grow without limit and destroy the aircraft. Where non-linearity occurs, a limit cycle oscillation (LCO) may be encountered. Flutter is clearly a dangerous phenomenon and must be treated very carefully, both by calculation and test.

In a flutter test, the idea is to progress through the flight envelope, gradually increasing velocity and Mach number in order to examine the change in aeroelastic stability. At each test point, the aircraft is excited in some way so that it vibrates, and the excitation and response signals are used to obtain estimates of the frequency and damping of all the modes that are active. The frequency and damping values are then plotted against velocity (or Mach number) and the trend of damping examined; any downward trend in damping can indicate the likely approach of flutter (which occurs at zero damping) and so increased care needs to be taken with subsequent tests.

A number of test signals may be used to excite the structure at each test point deliberately, typically impulse (or sequence of impulses), random or chirp (a sine wave with increasing frequency); the chirp is arguably the most popular excitation signal as it can yield a high signal-to-noise ratio. The signals may be implemented by use of an aerodynamic vane, an inertial exciter or, most commonly, a control surface (via the flight control system and control surface actuator). When using a special device to excite the structure (e.g. vane or inertial exciter), it is normal to use some measure of the excitation as a reference signal for computing the frequency response function (FRF), prior to estimating the parameters by some system identification approach [2]. In the case when the control surface is being used via a hydraulic (or other) actuator, it is most common to use the demand signal to the actuator as the reference signal for data processing.

However, the authors are aware from anecdotal and published evidence that sometimes a different reference signal is employed, namely the actuator force (or strain) or the measured control angle (or actuator displacement). Also, published references often do not make clear what signal is actually being used as a reference for the excitation. A limited number of examples of where other signals appear to have been used will now be given. In the AGARD Flight Instrumentation Series [3] measurements are made of the 'actual input force' associated with use of a servo actuator. In reference [4], the term 'control deflection signals' is used for flutter testing of a drone with the active flutter suppression system off. In reference [5], the 'aileron rotational position' was used for transfer function analysis, but only in a qualitative sense to confirm results from sine dwell tests, as it was recognized that this signal was not a 'true force reference'. In reference [6], 'aileron motion sensors' are employed for computing the frequency response functions (FRFs).

In this paper, it is shown via a mathematical model of a wing/control surface with a simple actuator that using either control angle or control force as a reference is incorrect and constitutes bad practice. It can even lead to a dangerous situation where the onset of flutter is not seen in the test results. It is therefore con-sidered essential to use the demand signal as a reference and publications on flutter testing should always be explicit about what reference signal was employed.

\section{BASIC WING/CONTROL MODEL}

\subsection{Structural terms}

The simple model chosen for this paper is a 3 degree-offreedom system consisting of a rigid wing flapping with 


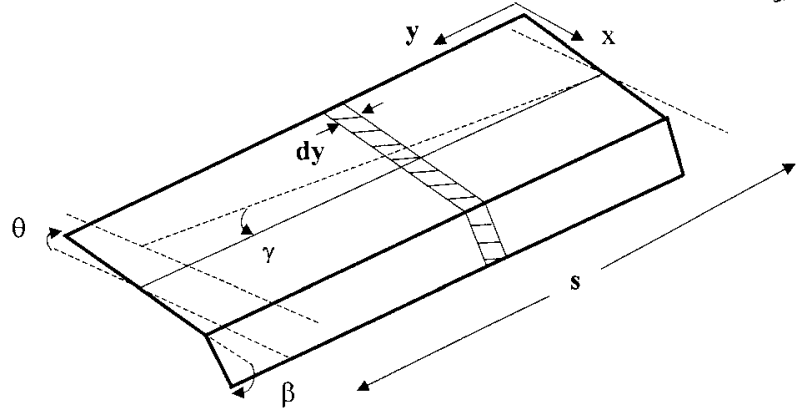

Fig. 1 Schematic layout of wing/control surface deformations

an angle $\gamma$ about a hinge at the root, twisting with an angle $\theta$ and with a rigid control surface rotating through an angle $\beta$, as shown in Figs 1 and 2. The model is based on that used in reference [7], albeit augmented to include a control surface. In inertial terms, the wing and control surface are considered to be of uniform thickness and density but aerodynamically the model would have a conventional aerofoil section. The rotational motions are constrained by discrete springs of stiffness $k_{\gamma}, k_{\theta}$ and $k_{\beta}$ respectively, such that there are no stiffness coupling terms. The total potential energy of the system, $U$, can then be expressed as

$$
U=\frac{1}{2} k_{\gamma} \gamma^{2}+\frac{1}{2} k_{\theta} \theta^{2}+\frac{1}{2} k_{\beta} \beta^{2}
$$

The total kinetic energy of the system, $T$, can be expressed as

$$
\begin{aligned}
T= & \frac{1}{2} \int_{0}^{s} \int_{0}^{x_{\mathrm{h}}} \mathrm{d} m\left[\dot{\gamma} y+\left(x-x_{\mathrm{f}}\right) \dot{\theta}\right]^{2}+\frac{1}{2} \int_{0}^{s} \int_{x_{\mathrm{h}}}^{c} \mathrm{~d} m \\
& \times\left\{\left[\dot{\gamma} y+\left(x-x_{\mathrm{f}}\right) \dot{\theta}\right]+\left(x-x_{\mathrm{h}}\right) \dot{\beta}\right\}^{2}
\end{aligned}
$$

where the 'overdot' refers to $\mathrm{d} / \mathrm{d} t, \mathrm{~d} m$ refers to an elemental mass, $s$ is the span, $c$ is the chord, $x_{\mathrm{f}}$ defines the position of the flexural axis, $x_{\mathrm{h}}$ defines the hinge line position and the two integral terms refer to the main wing surface and control surface respectively.

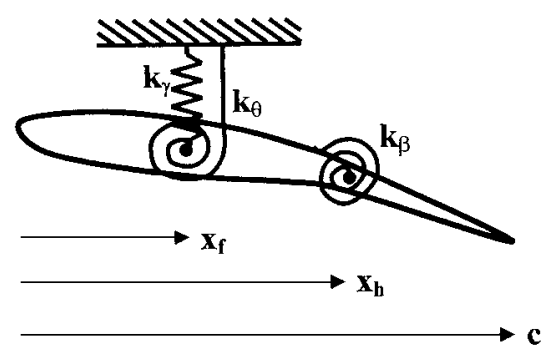

Fig. 2 Schematic layout of the wing/control surface with spring
Applying Lagrange's equations to the energy terms in equations (1) and (2) leads to the expression

$$
\begin{aligned}
& {\left[\begin{array}{ccc}
I_{\gamma} & I_{\gamma \theta} & I_{\gamma \beta} \\
\text { symmetry } & I_{\theta} & I_{\theta \beta} \\
I_{\beta}
\end{array}\right]\left\{\begin{array}{l}
\gamma \\
\theta \\
\beta
\end{array}\right\}+\left[\begin{array}{ccc}
k_{\gamma} & 0 & 0 \\
0 & k_{\theta} & 0 \\
0 & 0 & k_{\beta}
\end{array}\right]\left\{\begin{array}{l}
\gamma \\
\theta \\
\beta
\end{array}\right\}} \\
& =\left\{\begin{array}{l}
Q_{\gamma} \\
Q_{\theta} \\
Q_{\beta}
\end{array}\right\}
\end{aligned}
$$

Here, $Q_{\gamma}, Q_{\theta}$ and $Q_{\beta}$ are generalized forces due to aerodynamic effects. Also, $I_{\gamma}, I_{\theta}$ and $I_{\beta}$ are moments of inertia and $I_{\gamma \theta}, \quad I_{\gamma \beta}$ and $I_{\theta \beta}$ are product moments of inertia, all taken about the relevant axes of rotation and defined as

$$
\begin{aligned}
& I_{\gamma}=\iint_{w+c} y^{2} \mathrm{~d} m \\
& I_{\theta}=\iint_{w+c}\left(x-x_{\mathrm{f}}\right)^{2} \mathrm{~d} m \\
& I_{\beta}=\iint_{c}\left(x-x_{\mathrm{h}}\right)^{2} \mathrm{~d} m \\
& I_{\gamma \theta}=\iint_{w+c} y\left(x-x_{\mathrm{f}}\right) \mathrm{d} m \\
& I_{\gamma \beta}=\iint_{c} y\left(x-x_{\mathrm{h}}\right) \mathrm{d} m \\
& I_{\theta \beta}=\iint_{c}\left(x-x_{\mathrm{f}}\right)\left(x-x_{\mathrm{h}}\right) \mathrm{d} m
\end{aligned}
$$

where integrals are taken over the wing plus control surface $(w+c)$ or the control surface alone $(c)$.

\subsection{Aerodynamic terms}

The generalized force is defined by $Q_{i}=\partial(\delta W) / \partial q_{i}$, where $q_{i}$ is a generalized coordinate (i.e. $q_{1,2,3}$ correspond to $\gamma, \theta$ and $\beta$ respectively) and $\delta W$ is the incremental work done when an incremental change of generalized coordinates occurs. This equation can now be used to calculate the aerodynamic forces acting on a strip of width $\mathrm{d} y$, as shown in Fig. 1. The generalized forces $Q_{\gamma}$, $Q_{\theta}$ and $Q_{\beta}$ correspond to unsteady aerodynamic forces and are a function of the frequency parameter

$$
v=\frac{\omega c}{V}
$$

where $c$ is the chord length, $\omega$ is the frequency and $V$ is the relative velocity. However, for simplicity, the quasisteady form of the aerodynamics will be used to allow the forces and moments to be expressed in terms of angles and angular velocities*. However, the quasi-steady

\footnotetext{
* Quasi-steady aerodynamics neglect the attenuation and phase lag associated with an increase in the frequency of oscillation and are defined as the limit of unsteady oscillatory aerodynamics as the frequency parameter tends to zero. Here, the quasi-steady assumption is that the aerodynamic characteristics of an aerofoil undergoing variable motions are equal, at any instant of time, to the characteristics of the same aerofoil moving with constant velocities equal to the instantaneous values.
} 
aerodynamics are modified to include the non-dimensional aerodynamic torsional damping derivative, $M_{\dot{\theta}}$, as suggested in reference [7], to yield a more realistic flutter phenomenon for the quasi-steady case; a control aerodynamic damping derivative, $M_{\dot{\beta}}$, is also included. The quasi-steady assumption, while not yielding accurate flutter predictions, does not affect the arguments made herein about the different approaches to flutter testing and is simple to derive for this basic model.

Applying two-dimensional 'strip theory' results for the wing/control surface leads to the lift and pitching moment (positive leading edge up) about the flexural axis for a strip $\mathrm{d} y$ as

$$
\begin{aligned}
& \mathrm{d} L=\frac{1}{2} \rho V^{2} c \mathrm{~d} y\left[a\left(\theta+\frac{\dot{\gamma} y}{V}\right)+a_{\mathrm{c}} \beta\right] \\
& \mathrm{d} M=\frac{1}{2} \rho V^{2} c^{2} \mathrm{~d} y\left[a e\left(\theta+\frac{\dot{\gamma} y}{V}\right)+a_{\mathrm{m}} \beta+M_{\dot{\theta}} \frac{\dot{\theta} c}{V}\right]
\end{aligned}
$$

where $\dot{\gamma} y$ is the effective 'heave' velocity of the strip, $a_{\mathrm{c}}=\partial C_{\mathrm{L}} / \partial \beta, a_{\mathrm{m}}=\partial C_{\mathrm{M}} / \partial \beta, a$ is the two-dimensional lift curve slope, $e c$ is the eccentricity (distance of the flexural axis aft of the aerodynamic centre) and $M_{\dot{\theta}}<0$. The aerodynamic hinge moment on the strip (based on the wing chord and positive trailing edge down) can be shown to be

$$
\mathrm{d} H=\frac{1}{2} \rho V^{2} c^{2} \mathrm{~d} y\left[b_{1}\left(\theta+\frac{\dot{\gamma} y}{V}\right)+b_{2} \beta+M_{\dot{\beta}} \frac{\dot{\beta} c}{V}\right]
$$

where $b_{1}=\partial C_{\mathrm{H}} / \partial \alpha, \quad b_{2}=\partial C_{\mathrm{H}} / \partial \beta$ and $M_{\dot{\beta}}<0$. The incremental work done on the wing/control, $\delta W_{\text {aero }}$, by aerodynamic forces $\mathrm{d} L, \mathrm{~d} M$ and $\mathrm{d} H$ is

$$
\delta W_{\text {aero }}=-\int_{0}^{s} \mathrm{~d} L(y \delta \gamma)+\int_{0}^{s} \mathrm{~d} M(\delta \theta)+\int_{0}^{s} \mathrm{~d} H(\delta \beta)
$$

where $\delta \gamma, \delta \theta$ and $\delta \beta$ are the incremental changes in angles associated with $\mathrm{d} L, \mathrm{~d} M$ and $\mathrm{d} H$ respectively. The aerodynamic matrices will be derived in section 2.4.

\subsection{External excitation}

However, in addition, a way to input an excitation force to the wing model is needed for the flutter test to be performed. Therefore, using the geometry of the wing, the incremental work done by a direct force acting on the wing main surface or control surface can be obtained as

$$
\begin{aligned}
\delta W_{\text {direct force }}= & F_{\mathrm{m}}\left[y_{\mathrm{m}} \delta \gamma+\left(x_{\mathrm{m}}-x_{\mathrm{f}}\right) \delta \theta\right] \\
& +F_{\mathrm{c}}\left[y_{\mathrm{c}} \delta \gamma+\left(x_{\mathrm{c}}-x_{\mathrm{f}}\right) \delta \theta+\left(x_{\mathrm{c}}-x_{\mathrm{h}}\right) \delta \beta\right]
\end{aligned}
$$

where the subscripts $\mathrm{m}$ and $\mathrm{c}$ refer to the main and control surfaces respectively.

\subsection{Aeroelastic equations}

The full incremental work term $\delta W_{\text {total }}$ is therefore

$$
\delta W_{\text {total }}=\delta W_{\text {aero }}+\delta W_{\text {direct force }}
$$

The generalized forces can be calculated by differentiating the incremental work terms defined in equations (9) and (10) by the corresponding incremental rotations. Combining these results with that obtained from equation (3) yields the full aeroelastic equations

$$
\begin{aligned}
& {\left[\begin{array}{ccc}
I_{\gamma} & I_{\gamma \theta} & I_{\gamma \beta} \\
\text { symmetric } & I_{\theta} & I_{\theta \beta} \\
I_{\beta}
\end{array}\right]\left\{\begin{array}{c}
\gamma \\
\theta \\
\beta
\end{array}\right\}} \\
& +\frac{1}{2} \rho V s\left[\begin{array}{ccc}
\frac{a s^{2}}{3} & 0 & 0 \\
-\frac{a e s c}{2} & c^{2} M_{\dot{\theta}} & 0 \\
-\frac{b_{1} s c}{2} & 0 & -c^{2} M_{\dot{\beta}}
\end{array}\right]\left\{\begin{array}{c}
\dot{\gamma} \\
\dot{\theta} \\
\dot{\beta}
\end{array}\right\} \\
& +\frac{1}{2} \rho V^{2} s\left[\begin{array}{ccc}
0 & \frac{a s}{2} & \frac{a_{\mathrm{c}} s}{2} \\
0 & -a e c & -a_{\mathrm{m}} c \\
0 & -b_{1} c & -b_{2} c
\end{array}\right]\left\{\begin{array}{l}
\gamma \\
\theta \\
\beta
\end{array}\right\} \\
& \left.+\left[\begin{array}{ccc}
k_{\gamma} & 0 \\
0 & k_{\theta} & 0 \\
0 & 0 & k_{\beta}
\end{array}\right]\left\{\begin{array}{c}
\gamma \\
\theta \\
\beta
\end{array}\right\} \begin{array}{c}
y_{\mathrm{c}} \\
y_{\mathrm{m}} \\
x_{\mathrm{m}}-x_{\mathrm{f}} \\
0
\end{array}\right\}+F_{\mathrm{c}}\left\{\begin{array}{c}
x_{\mathrm{c}}-x_{\mathrm{f}} \\
x_{\mathrm{c}}-x_{\mathrm{h}}
\end{array}\right\}
\end{aligned}
$$

This matrix equation can be written in the classical form as follows:

$$
[\mathbf{A}] \boldsymbol{q}+(\rho V[\mathbf{B}]+[\mathbf{D}]) \dot{\boldsymbol{q}}+\left(\rho V^{2}[\mathbf{C}]+[\mathbf{E}]\right) \boldsymbol{q}=\boldsymbol{F}
$$

where $[\mathbf{A}]$ is the inertia matrix, $[\mathbf{B}]$ is the aerodynamic damping matrix, $[\mathbf{D}]$ is the structural damping matrix, $[\mathbf{C}]$ is the aerodynamic stiffness matrix, $[\mathbf{E}]$ is the structural stiffness matrix, $\boldsymbol{F}$ is the external forcing vector and $\boldsymbol{q}=\{\gamma, \theta, \beta\}^{\mathrm{T}}$ is the vector of generalized coordinates. The structural damping has been ignored in equation (12) in order to gain conservative answers, but the aerodynamic damping terms usually tend to dominate anyway.

\subsection{Flutter solution}

In order to determine the exact flutter characteristics of the model to use for evaluation of a simulated flutter test, a classical eigenvalue approach needs to be adopted. Firstly, the excitation force $\boldsymbol{F}$ is set to zero for free vibration and then a solution for the generalized 
response is taken as

$$
\boldsymbol{q}=\boldsymbol{q}_{0} \mathrm{e}^{\lambda t}
$$

where $\boldsymbol{q}_{0}$ is the response amplitude, $t$ is time and $\lambda$ is the exponent that dictates the stability of the system as velocity $V$ increases. Substituting this response into equation (13) yields a complex eigenvalue problem

$$
\left[\lambda^{2}[\mathbf{A}]+\lambda(\rho V[\mathbf{B}]+[\mathbf{D}])+\left(\rho V^{2}[\mathbf{C}]+[\mathbf{E}]\right)\right] \boldsymbol{q}_{0}=\mathbf{0}
$$

but this is normally solved by transforming to first-order form. The resulting eigenvalues are then of the form $\lambda_{j}=\mu_{j} \pm \mathrm{i} \omega_{j}(j=1,2,3)$ and may be used to determine the frequency and damping of the three roots of this system. Parameters for the model (see Appendix 1) were chosen to cause a 'control surface flutter', i.e. one involving control surface rotation; this introduces the complexity of simultaneously using the control surface for excitation while it is active in the flutter mechanism. A plot of the frequency and damping values against velocity is shown in Fig. 3a, where it may be seen that the flutter mechanism involves bending and control rotation and that the approximate flutter velocity is $40 \mathrm{~m} / \mathrm{s}$. Note that the modes tend to couple as the flutter velocity is approached so descriptions of bending, etc., become less meaningful.

\subsection{Simulated flutter test}

In order to demonstrate that a flutter test carried out using a conventional excitation force applied to the wing or control surface is able to predict the flutter velocity, a simulated test was carried out on the model in equation (12). A SIMULINK model was developed and a chirp excitation was applied to the wing surface, moving at a velocity of $30 \mathrm{~m} / \mathrm{s}$, so yielding the typical response at the excitation point shown in Fig. 4. The FRF between the response and excitation at the excitation point was calculated and the rational fraction polynomial identification method [8] was used to estimate the frequency and damping values for the system at this velocity. Table 1 shows good agreement between the results, so confirming accurate simulations.

Table 1 Comparison of theoretical and estimated values at $30 \mathrm{~m} / \mathrm{s}$

\begin{tabular}{llll}
\hline $\begin{array}{l}\text { Theoretical } \\
\text { frequencies } \\
(\mathrm{Hz})\end{array}$ & $\begin{array}{l}\text { Estimated } \\
\text { frequencies } \\
(\mathrm{Hz})\end{array}$ & $\begin{array}{l}\text { Theoretical } \\
\text { dampings } \\
(\%)\end{array}$ & $\begin{array}{l}\text { Estimated } \\
\text { dampings } \\
(\%)\end{array}$ \\
\hline 1.8793 & 1.8836 & 0.3625 & 0.3689 \\
2.4570 & 2.4551 & 0.6254 & 0.6311 \\
9.1130 & 9.1147 & 0.2411 & 0.2397 \\
\hline
\end{tabular}

The process was repeated at a range of velocities and the results for the simulated test are shown in Fig. $3 b$ to follow the exact results and so be able to predict the flutter velocity adequately. A similar result occurred for an excitation force applied on the control surface. Thus a measured excitation applied to the wing or control surface (e.g. inertia exciter, bonker, vane, etc.) is satisfactory for flutter testing provided the applied force is measured or some characteristics of the force are assumed (e.g. a flat spectrum). For these excitation types, the excitation signal is independent of the response and so does not interfere with the flutter characteristics.

\section{WING/CONTROL MODEL WITH ACTUATOR}

In order to examine the use of an actuator (e.g. hydraulic) to drive a control surface by providing the excitation stimulus for a flutter test, a modified model needs to be developed where the rotational spring $k_{\beta}$ is replaced by an actuator as shown in Fig. 5. The actuator chosen is a hydraulic device with pressure feedback stabilization, as shown in Fig. 6. The flow equations will be linearized in order to allow the exact flutter characteristics of the linear model to be determined. The type of actuator employed is considered to be immaterial to the results of this paper, provided that the equations may be linearized. The impact of using a nonlinear actuator in flutter testing is not addressed in this paper, but it is believed that using the actuator as an excitation device may well affect the flutter behaviour.

\subsection{Actuator model}

The piston is assumed to be at the centre of its stroke, and the external load and input demand are assumed to be small and oscillatory. Thus the pressure difference between the chambers is

$$
P_{\mathrm{J}}=P_{1}-P_{2}
$$

where $P_{1,2}$ are the pressures in each chamber and any displacements of the valve or piston are small. Also, the inertia force associated with the mass of the actuator body is assumed to be small for the frequency range of interest.

The valve opening $X_{\mathrm{V}}$ will be given by

$$
X_{\mathrm{V}}=\mu\left(X_{i}-X_{0}\right)-P_{\mathrm{J}} \frac{A_{\mathrm{F}}}{K_{\mathrm{F}}}
$$

where $\mu$ is the mechanical gearing, $X_{i}$ is the demanded displacement, $X_{0}$ is the displacement of the actuator body and $A_{\mathrm{F}}$ and $K_{\mathrm{F}}$ are the area and stiffness of the pressure feedback stabilization components. The flows 


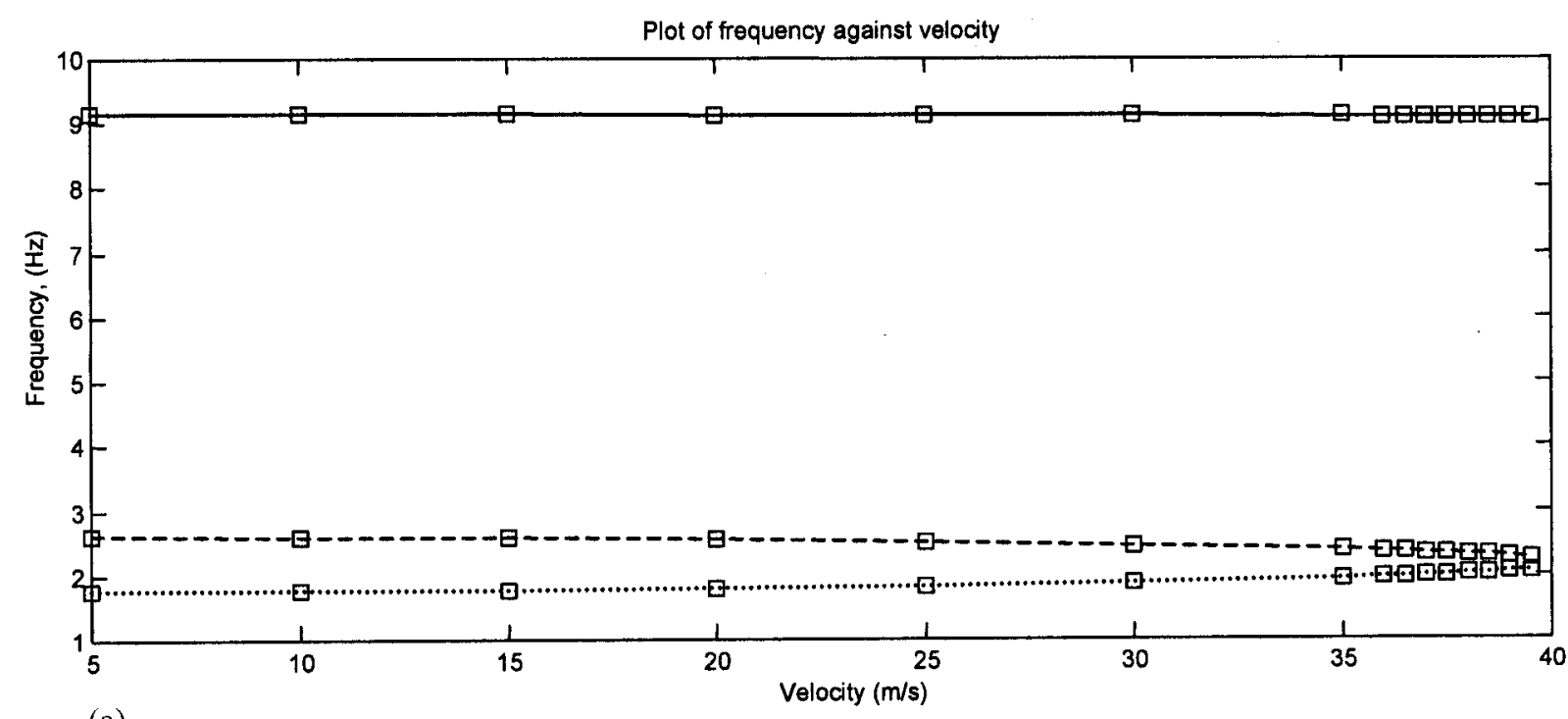

(a)

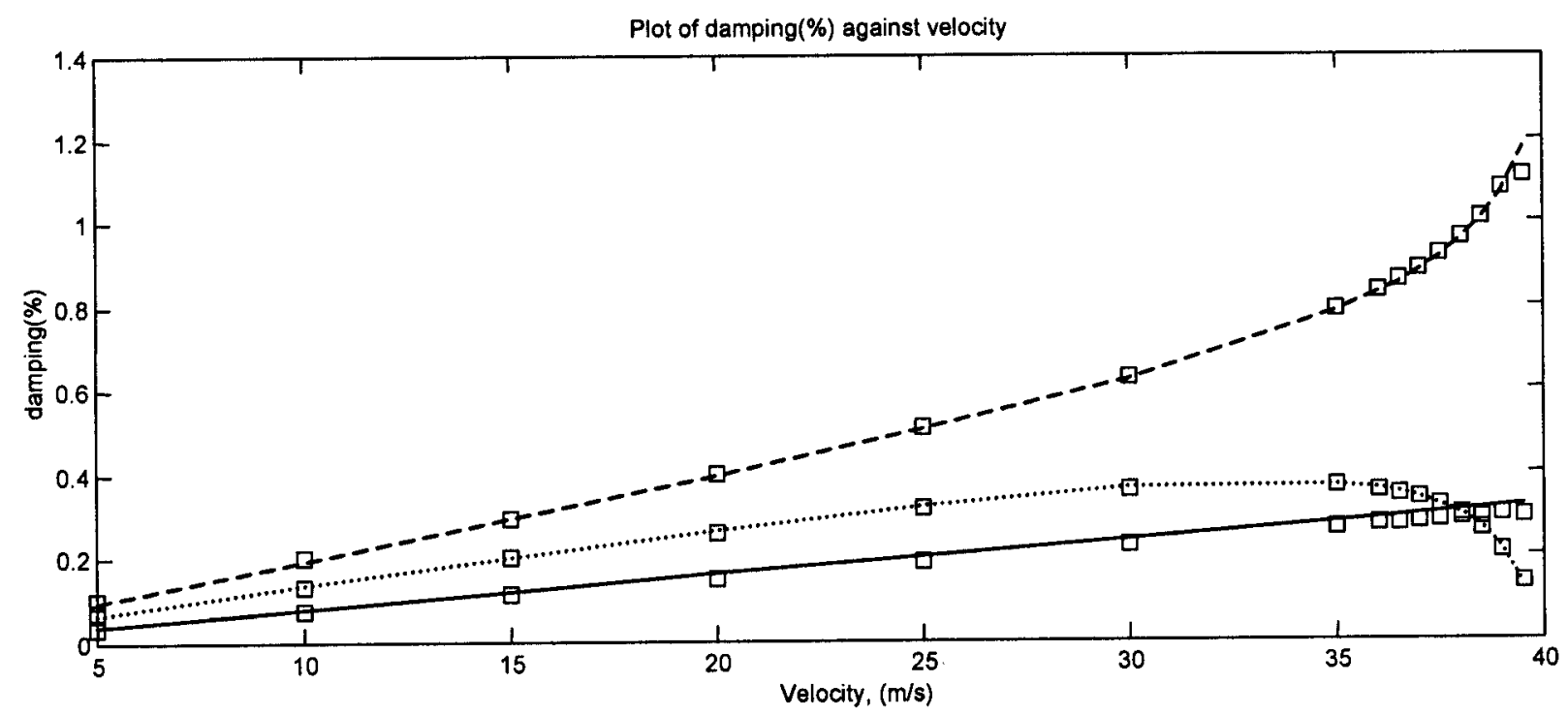

(b)

Fig. 3 Variation of frequency and damping against velocity for the basic wing/control surface model: (a) solid line, exact values from the flutter solution; (b) points, estimated values from the simulated flutter test

$Q_{1,2}$ into and out of the chambers through the valves are

$$
\begin{array}{rr}
Q_{1}=K_{\mathrm{V}} \sqrt{P_{\mathrm{S}}-P_{1}} X_{\mathrm{V}}, & Q_{2}=K_{\mathrm{V}} \sqrt{P_{2}-P_{\mathrm{R}}} X_{\mathrm{V}} \\
& \text { for } X_{\mathrm{V}} \geqslant 0 \\
Q_{1}=K_{\mathrm{V}} \sqrt{P_{1}-P_{\mathrm{R}}} X_{\mathrm{V}}, & Q_{2}=K_{\mathrm{V}} \sqrt{P_{\mathrm{S}}-P_{2}} X_{\mathrm{V}} \\
\text { for } X_{\mathrm{V}}<0
\end{array}
$$

where $K_{\mathrm{V}}$ is the valve flow constant and $P_{\mathrm{R}}$ and $P_{\mathrm{S}}$ are the return and supply pressures respectively. The expressions are clearly non-linear in the pressures. For small oscillatory loads and displacements, and with the return pressure assumed to be negligible $\left(P_{\mathrm{R}}=0\right)$, there will be a small oscillation of the pressures about $P_{\mathrm{S}} / 2$. Thus, equations (18) can be linearized as

$$
Q_{1}=Q_{2}=K_{\mathrm{V}} \sqrt{\frac{P_{\mathrm{S}}}{2}} X_{\mathrm{V}}
$$

without losing the basic flow mechanism that is controlled by the valve motion. The flow through the valve must balance the rate of change of the oil volume $V_{\mathrm{o}}$ (i.e. $V_{\mathrm{o}} / 2$ per chamber) due to compression of the oil 


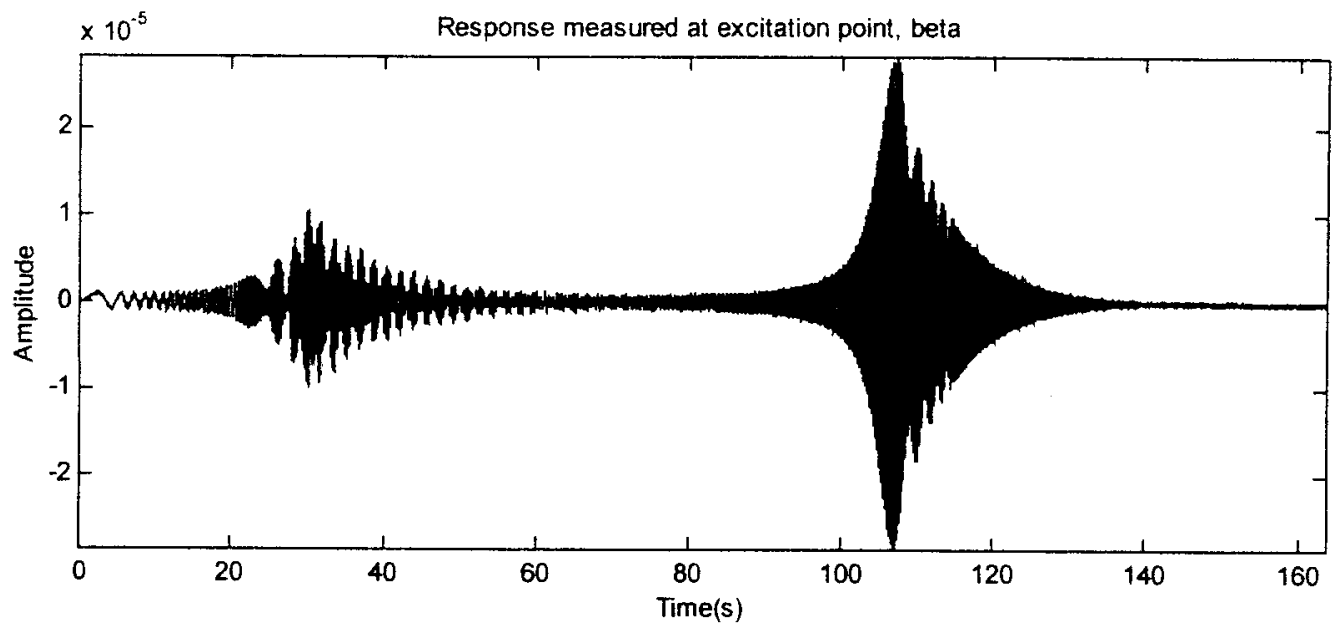

Fig. 4 Sample response to a chirp at $30 \mathrm{~m} / \mathrm{s}$ velocity for the wing/control model

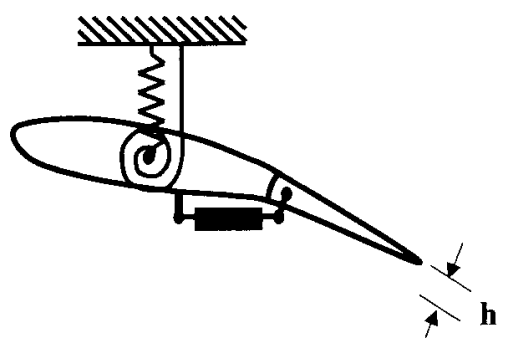

Fig. 5 Schematic layout of the wing/control surface with actuator

and due to piston movement, so that

$$
\begin{aligned}
& Q_{1}=\frac{V_{\mathrm{o}}}{2 N} \dot{P}_{1}+A_{\mathrm{P}} \dot{X}_{0} \\
& Q_{2}=-\frac{V_{\mathrm{o}}}{2 N} \dot{P}_{2}+A_{\mathrm{P}} \dot{X}_{0}
\end{aligned}
$$

where leakage across the piston and flow into the pressure stabilization sensing chambers are neglected. Adding these equations together and using equation (19) gives

$$
2 K_{\mathrm{V}} \sqrt{\frac{P_{\mathrm{S}}}{2}} X_{\mathrm{V}}=\frac{V_{\mathrm{o}}}{2 N}\left(\dot{P}_{1}-\dot{P}_{2}\right)+2 A_{\mathrm{P}} \dot{X}_{0}
$$

which, after substituting for $P_{\mathrm{J}}$ from equation (16) and $X_{\mathrm{V}}$ from equation (17), gives a first-order differential equation for the pressure difference $P_{\mathrm{J}}$ :

$$
\begin{aligned}
& \frac{V_{\mathrm{o}}}{4 N} \dot{P}_{\mathrm{J}}+\frac{K_{\mathrm{V}} A_{\mathrm{F}}}{K_{\mathrm{F}}} \sqrt{\frac{P_{\mathrm{S}}}{2}} P_{\mathrm{J}} \\
& \quad=-A_{\mathrm{P}} \dot{X}_{0}-\mu K_{\mathrm{V}} \sqrt{\frac{P_{\mathrm{S}}}{2}} X_{0}+\mu K_{\mathrm{V}} \sqrt{\frac{P_{\mathrm{S}}}{2}} X_{i}
\end{aligned}
$$

Finally, the relationship between the force $F_{\mathrm{P}}$ applied to the actuator and the differential pressure is

$$
F_{\mathrm{P}}=-A_{\mathrm{P}} P_{\mathrm{J}}
$$

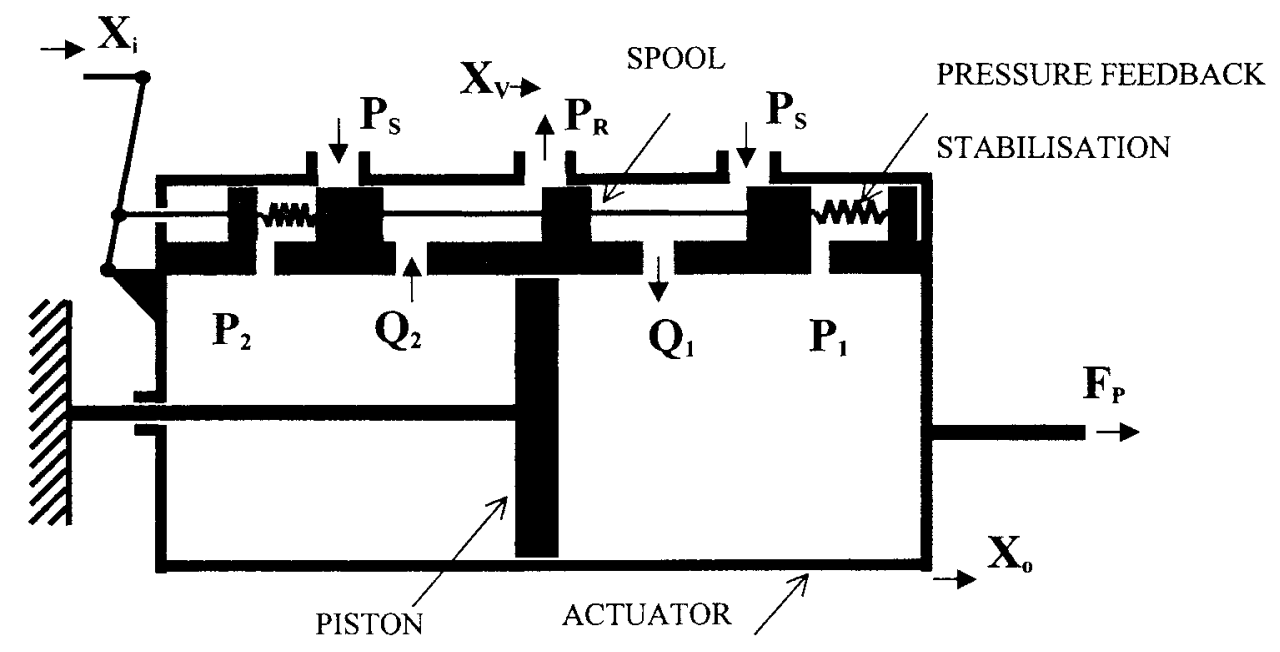

Fig. 6 Schematic layout of the actuator 
In Appendix 2, the characteristics of the actuator are examined further.

\subsection{Coupling the actuator into the wing/control equations}

Having found equations for the actuator, the two sets of equations must now be coupled using the kinematic relationship between the control angle $\beta$ and the actuator displacement $X_{0}$, namely

$$
X_{0}=-\beta h
$$

where $h$ is the lever arm for the actuator/control surface connection seen in Fig. 5. Thus

$$
\dot{X}_{0}=-\dot{\beta} h
$$

and

$$
X_{i}=-\beta_{i} h
$$

where $\beta_{i}$ is the effective demanded control angle and $X_{i}$ is the demanded actuator displacement.

Because the control spring has been removed, the restoring torque from the spring $\left(k_{\beta} \beta\right)$ needs to be replaced by a torque $T_{\beta}$, now provided by the actuator, namely

$$
T_{\beta}=-F_{\mathrm{P}} h=h A_{\mathrm{P}} P_{\mathrm{J}}
$$

where $F_{\mathrm{P}}$ is the force applied to the actuator body, as shown in Fig. 6. Combining equations (12) and (22) and using the coupling equations (24) to (26) and (27) can be shown to yield the aeroelastic equations incorporating the actuator, namely

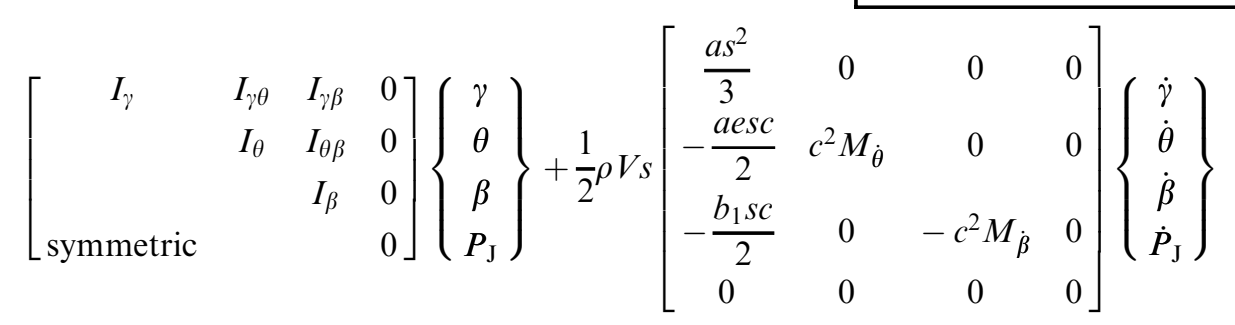

$$
\begin{aligned}
& +\left[\begin{array}{cccc}
0 & 0 & 0 & 0 \\
0 & 0 & 0 & 0 \\
0 & 0 & 0 & 0 \\
0 & 0 & -h A_{\mathrm{P}} & \frac{V_{\mathrm{o}}}{4 N}
\end{array}\right]\left\{\begin{array}{c}
\dot{\gamma} \\
\dot{\theta} \\
\dot{\beta} \\
\dot{P}_{\mathrm{J}}
\end{array}\right\}+\frac{1}{2} \rho V^{2} s\left[\begin{array}{cccc}
0 & \frac{a s}{2} & \frac{a_{\mathrm{c}} s}{2} & 0 \\
0 & -a e c & -a_{\mathrm{m}} c & 0 \\
0 & -b_{1} c & -b_{2} c & 0 \\
0 & 0 & 0 & 0
\end{array}\right]\left\{\begin{array}{c}
\gamma \\
\theta \\
\beta \\
P_{\mathrm{J}}
\end{array}\right\} \\
& +\left[\begin{array}{cccc}
k_{\gamma} & 0 & 0 & 0 \\
0 & k_{\theta} & 0 & 0 \\
0 & 0 & 0 & h A_{\mathrm{P}} \\
0 & 0 & -h \mu K_{\mathrm{V}} \sqrt{\frac{P_{\mathrm{S}}}{2}} & \frac{K_{\mathrm{V}} A_{\mathrm{F}}}{K_{\mathrm{F}}} \sqrt{\frac{P_{\mathrm{S}}}{2}}
\end{array}\right]\left\{\begin{array}{c}
\gamma \\
\theta \\
\beta \\
P_{\mathrm{J}}
\end{array}\right\}=\beta_{i}\left\{\begin{array}{c}
0 \\
0 \\
0 \\
-h \mu K_{\mathrm{V}} \sqrt{\frac{P_{\mathrm{S}}}{2}}
\end{array}\right\}
\end{aligned}
$$

The exact flutter solution for the wing/control with actuator may be found by a similar approach to that in section 2.4, where a first-order eigenvalue problem is set up to determine the roots of the system. In this case,

wing/control structure may be seen more clearly if equation (28) is rewritten compactly as

$$
\begin{aligned}
& {\left[\begin{array}{cc}
{[\mathbf{A}]} & \mathbf{0} \\
0 & 0
\end{array}\right]\left\{\begin{array}{c}
\boldsymbol{q} \\
P_{\mathrm{J}}
\end{array}\right\}+\left[\begin{array}{cc}
{[\rho V \mathbf{B}]} & \mathbf{0} \\
\mathbf{S} & \mathbf{R}
\end{array}\right]\left\{\begin{array}{c}
\dot{\boldsymbol{q}} \\
\dot{P}_{\mathrm{J}}
\end{array}\right\}} \\
& +\left[\begin{array}{cc}
{\left[\rho V^{2} \mathbf{C}+\mathbf{E}\right]} & \mathbf{T} \\
\mathbf{U} & \mathbf{W}
\end{array}\right]\left\{\begin{array}{c}
\boldsymbol{q} \\
P_{\mathrm{J}}
\end{array}\right\}=\left\{\begin{array}{c}
\mathbf{0} \\
\mathbf{Y}
\end{array}\right\} \beta_{i}
\end{aligned}
$$

where the matrices $[\mathbf{A}],[\mathbf{B}]$ and $[\mathbf{C}]$ are the same as shown earlier in equation (13), $[\mathbf{E}]$ is defined differently as

$$
[\mathbf{E}]=\left[\begin{array}{ccc}
k_{\gamma} & 0 & 0 \\
0 & k_{\theta} & 0 \\
0 & 0 & 0
\end{array}\right]
$$

and other actuator terms are introduced, namely

$$
\begin{aligned}
& \mathbf{T}=\left\{\begin{array}{c}
0 \\
0 \\
h A_{\mathrm{P}}
\end{array}\right\}, \quad \mathbf{S}=\left[\begin{array}{lll}
0 & 0 & -h A_{\mathrm{P}}
\end{array}\right], \quad \mathbf{R}=\frac{V_{\mathrm{o}}}{4 N} \\
& \mathbf{U}=\left[\begin{array}{lll}
0 & 0 & -h \mu K_{\mathrm{V}} \sqrt{\frac{P_{\mathrm{S}}}{2}}
\end{array}\right], \quad \mathbf{W}=\frac{K_{\mathrm{V}} A_{\mathrm{F}}}{K_{\mathrm{F}}} \sqrt{\frac{P_{\mathrm{S}}}{2}} \\
& \mathbf{Y}=-h \mu K_{\mathrm{V}} \sqrt{\frac{P_{\mathrm{S}}}{2}}
\end{aligned}
$$

\subsection{Flutter solution}

because the additional equation is only of first order, there will be three complex conjugate pairs as before, together with a real root that occurs because of the lag in the actuator frequency characteristics. The same
Note that the excitation force vector has now been removed because the actuator will provide the flutter test excitation via the control surface rotation. The coupling terms between the actuator and the 


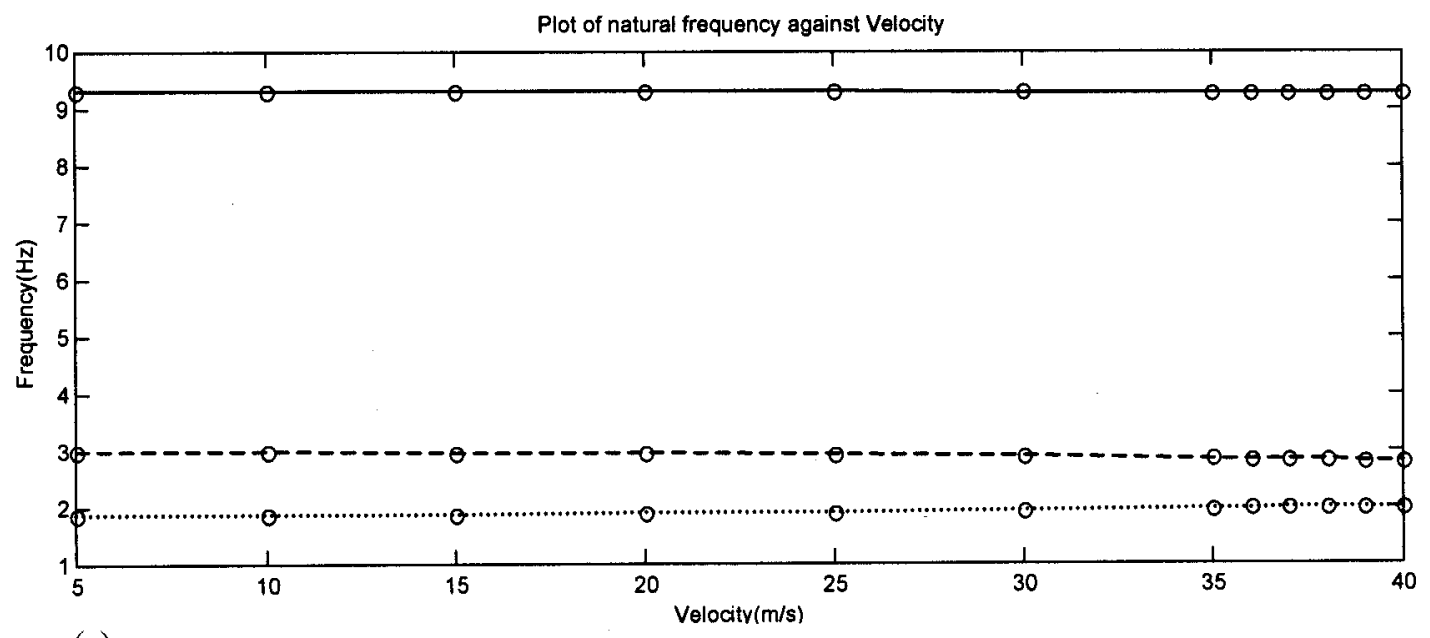

(a)

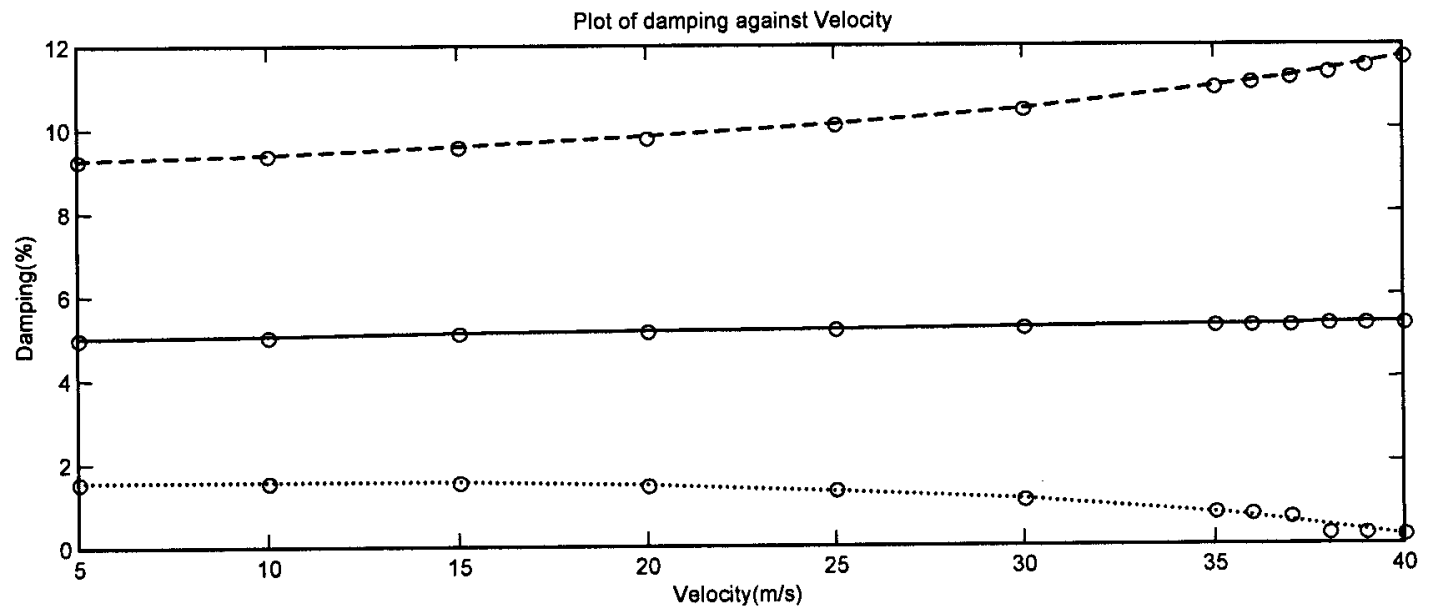

(b)

Fig. 7 Variation of frequency and damping against velocity for the wing/control surface with actuator model: (a) solid line, exact values from the flutter solution; (b) points, estimated values from the simulated flutter test. (- - ) angle of flap, $\gamma,(\ldots \ldots)$ control rotation angle, $\beta,(-)$ angle of twist, $\theta$

wing/control parameters were chosen as before but the actuator cannot exactly replace the control spring stiffness, $k_{\beta}$, because it is a far more complex system with frequency-dependent stiffness and damping. For this reason, the actuator parameters were chosen as discussed in Appendix 3. Once again, the aim was to achieve a 'control surface flutter'. A plot of the frequency and damping values against velocity for the wing/control surface with actuator model is shown in Fig. 7a, where it may be seen that the flutter mechanism involved bending and control rotation and that the approximate flutter velocity is $41 \mathrm{~m} / \mathrm{s}$, a value close to the earlier case. The flutter is 'softer' than before, i.e. the rate of change of damping with velocity as flutter is approached is more gradual. This is probably because of the additional actuator damping present in the control rotation. It should be noted that use of an actuator will in practice lead to a higher control frequency, but this model is simply being used to illustrate a point that is independent of the relative natural frequencies of the modes.

\subsection{Simulated flutter test}

In order to investigate how suitable the use of the control surface is for providing excitation in a flutter test with different reference signals used for estimating the FRF, a SIMULINK model was set up as before. Simulated flutter tests were performed at a series of 


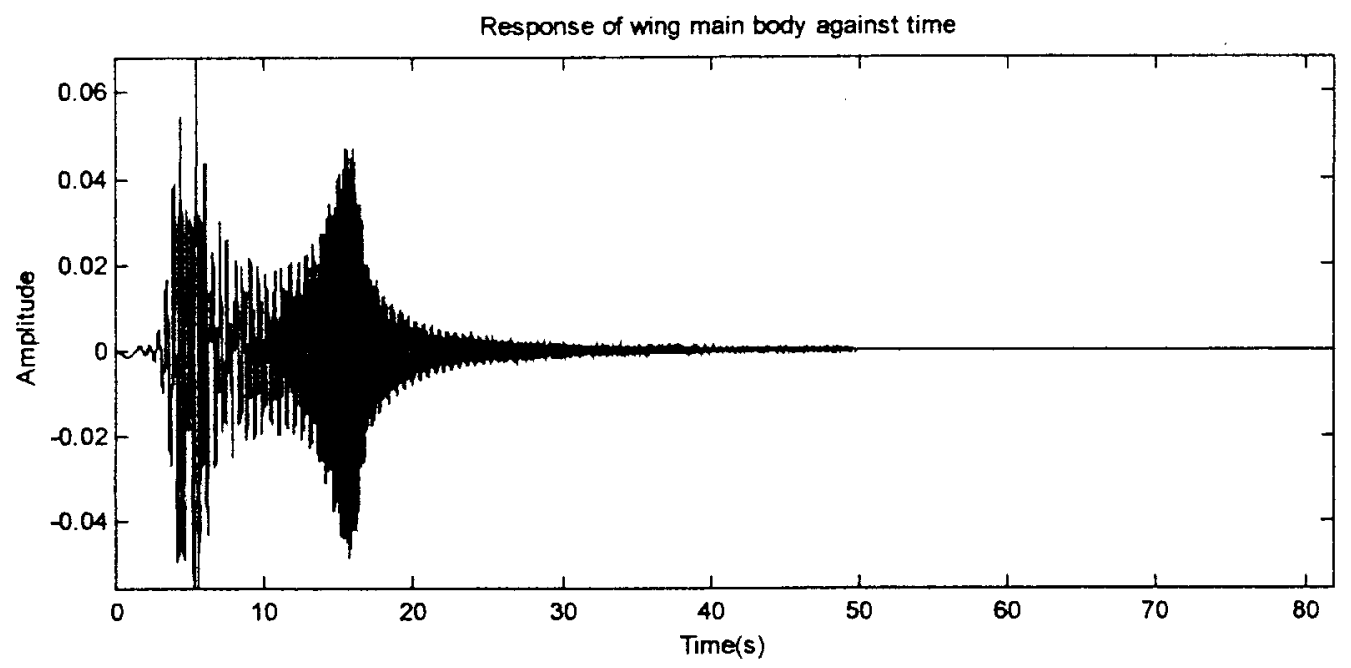

Fig. 8 Sample wing response to a chirp at $20 \mathrm{~m} / \mathrm{s}$ velocity for the wing/control with actuator model

velocities and with a chirp demand signal provided to the actuator input. FRFs were calculated between the response on the wing or control surface and one of several reference signals, as discussed below. The relevant FRFs were used together with the rational fraction polynomial method to identify the frequency and damping values at each test point.

Each of the following three reference signals was used in the simulated flutter test:

(a) the actuator demand signal itself,

(b) the force in the actuator/control link and

(c) the control rotation angle.

Sample time histories for the displacement response and reference signals at $20 \mathrm{~m} / \mathrm{s}$ are presented in Figs 8 to 10 . It may be noted immediately that the actuator force and control rotation angle reference signals actually reflect the dynamics of the system whereas obviously the demand signal does not, being generated using a constant amplitude chirp. FRFs corresponding to a selection of the response/reference combinations are presented in Figs 11 to 13. Figure 11, based upon the demand angle reference signal, shows peaks at the frequencies expected from the eigenvalue solution. However, it may be seen clearly in Figs 12 and 13, where the actuator force or control angle are used as reference signals, that the peak positions are not consistent with the true frequencies; a similar story applies for the FRFs based on a response on the control surface itself. Indeed, the peaks actually occur at the 'anti-resonances' of some of the other FRFs, as will be explained later. Finally, the FRF for the actuator force with respect to the demand angle shown in Fig. 14 demonstrates clearly that the actuator force actually contains the dynamics of the system; a similar result occurs for the control angle.

To complete the demonstration of the use of different reference signals, the results from the curve fit at a range of velocities is compared to the true flutter behaviour in Fig. $7 \mathrm{~b}$ for the wing response/control demand angle.

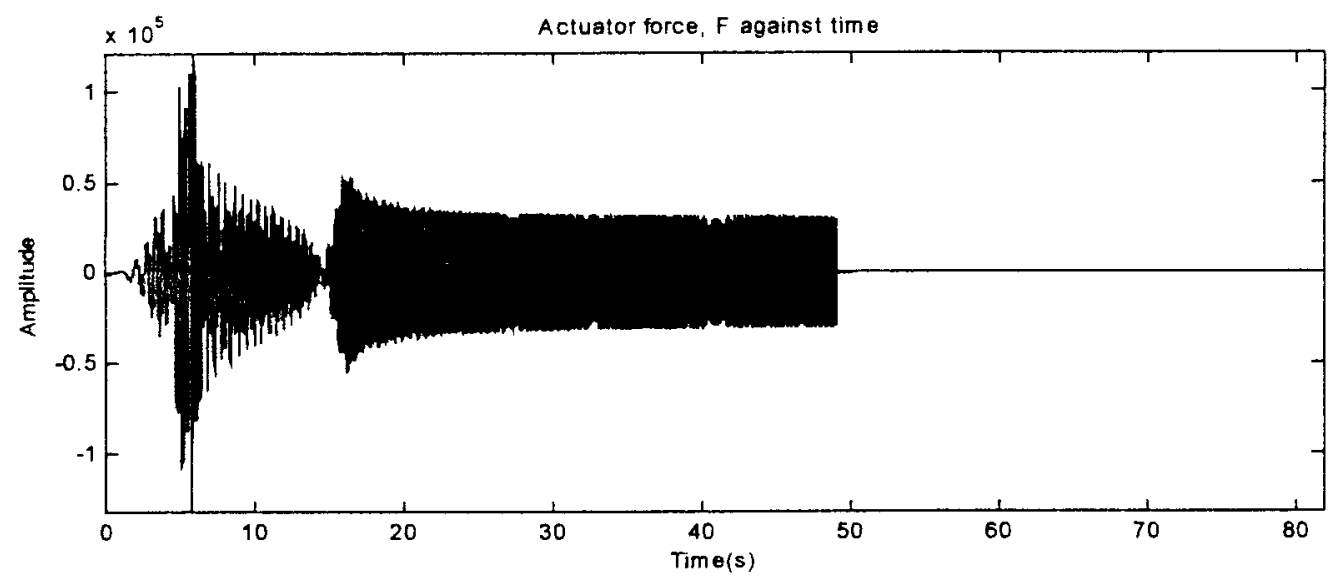

Fig. 9 Sample actuator force reference for a chirp excitation at $20 \mathrm{~m} / \mathrm{s}$ velocity for the wing/control with actuator model 


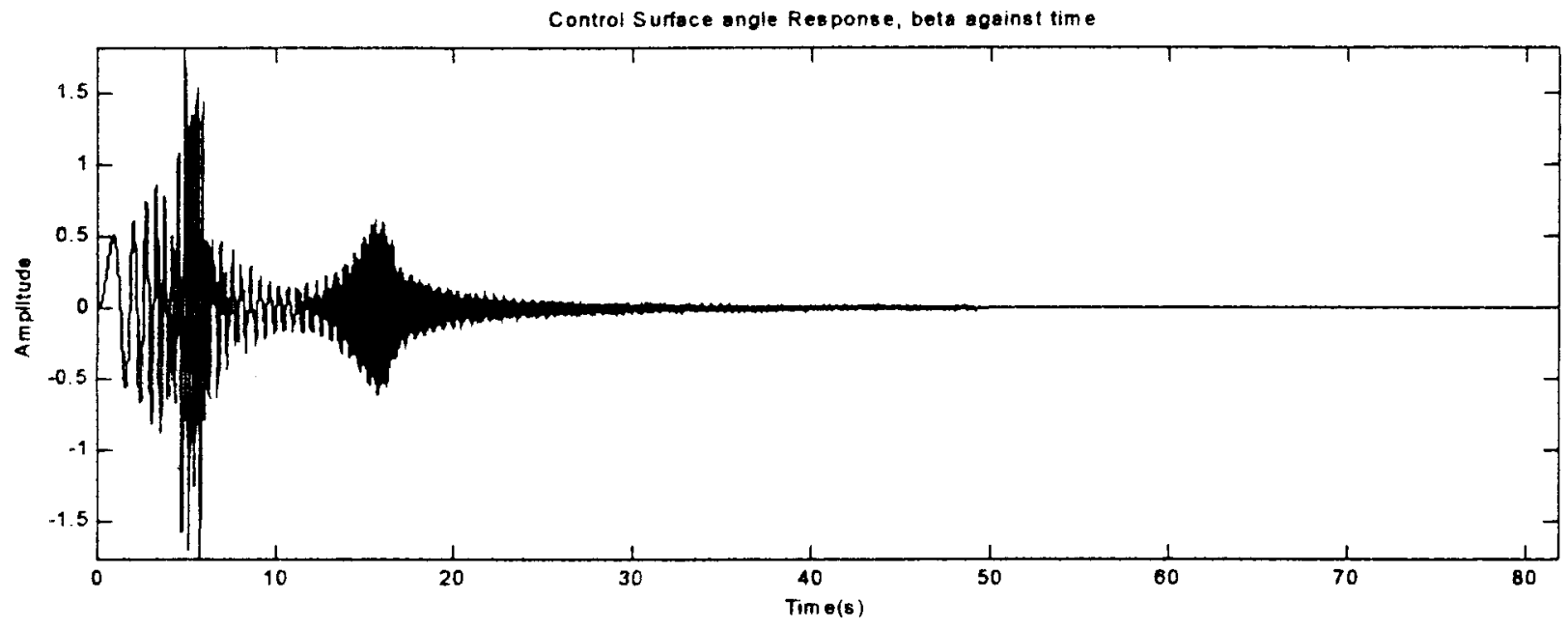

Fig. 10 Sample control angle reference for a chirp excitation at $20 \mathrm{~m} / \mathrm{s}$ velocity for the wing/control with actuator model

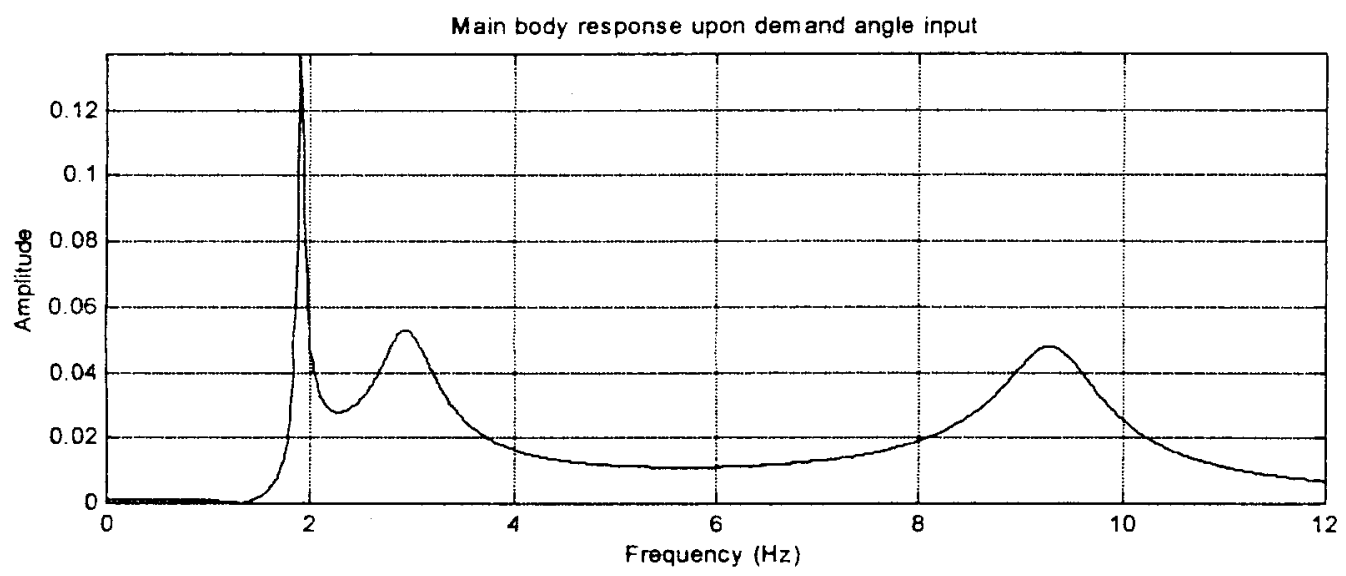

Fig. 11 Sample FRF between wing response and demand angle at $20 \mathrm{~m} / \mathrm{s}$ velocity for the wing/control with actuator model

The results compare very closely, so confirming accuracy of the simulations, and demonstrate that a satisfactory flutter test could be carried out for this reference signal. This result is repeated for all of the other FRFs using the control demand angle, even where the actuator force or control rotation are used as responses rather than references. This confirms that the actuator force and control rotation contain the essential response dynamics of the flutter system and should not be used as reference signals. It is also apparent that it is possible to use an actuator to provide excitation for a flutter test, even when it simultaneously acts as an impedance in the flutter model; it is not obvious that this is also the case when non-linearities are taken into account, which would require a separate study.

In order to confirm this conclusion, the FRFs in Figs 12 and 13, based upon using the actuator force or control rotation as reference signals, were curve-fitted, noting that in most cases there were only two clear peaks. The 'apparent' frequency values differed noticeably from the true frequencies, though sometimes the values were actually quite close. Also, the 'apparent' damping values did not indicate a flutter at all in the velocity range up to $40 \mathrm{~m} / \mathrm{s}$; nor was there any sign from the damping trend that flutter was imminent. In most cases the 'apparent' damping values increased with velocity or remained essentially constant, as shown in Figs 15 and 16, which use actuator force and control angle respectively as reference signals. In essence, pseudo 'modes' have been identified; even though the frequencies look quite close to the true values in this example, they are incorrect and so are the damping values.

Thus the use of the actuator force (or strain) or control rotation angle (or actuator displacement) as a reference signal in a flutter test is totally inappropriate and could be dangerous, misleading the analyst to believe that there was no flutter problem when in fact a flutter could be imminent. The only suitable reference 


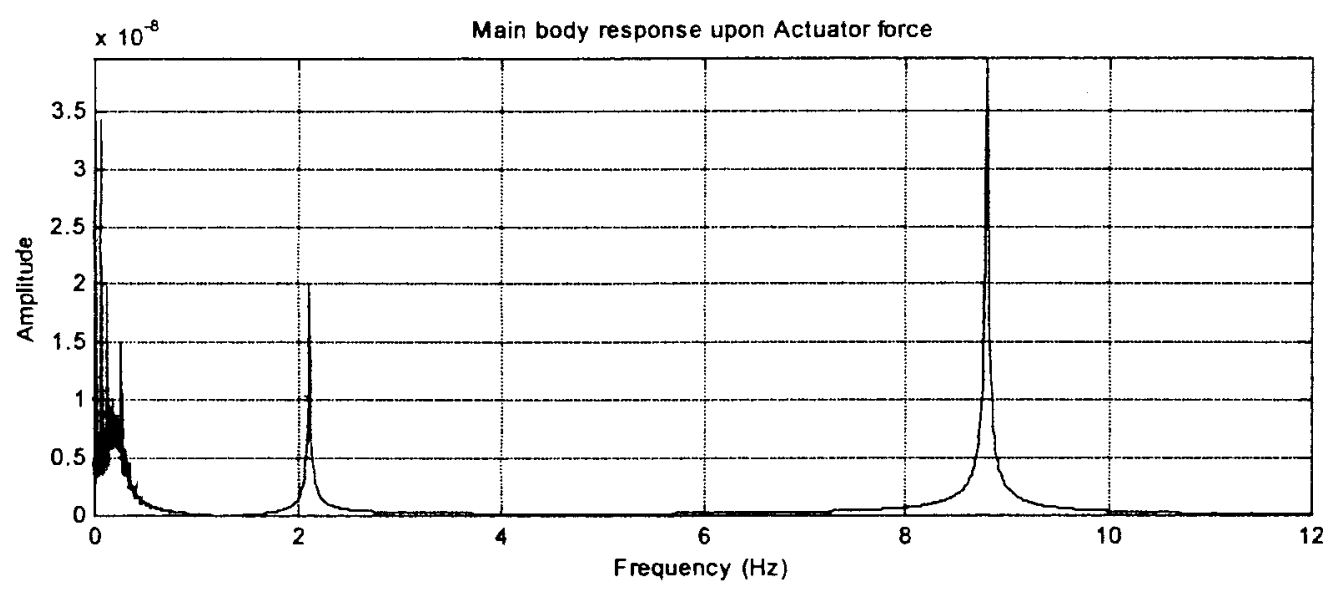

Fig. 12 Sample FRF between wing response and actuator force at $20 \mathrm{~m} / \mathrm{s}$ velocity for the wing/control with acutator model

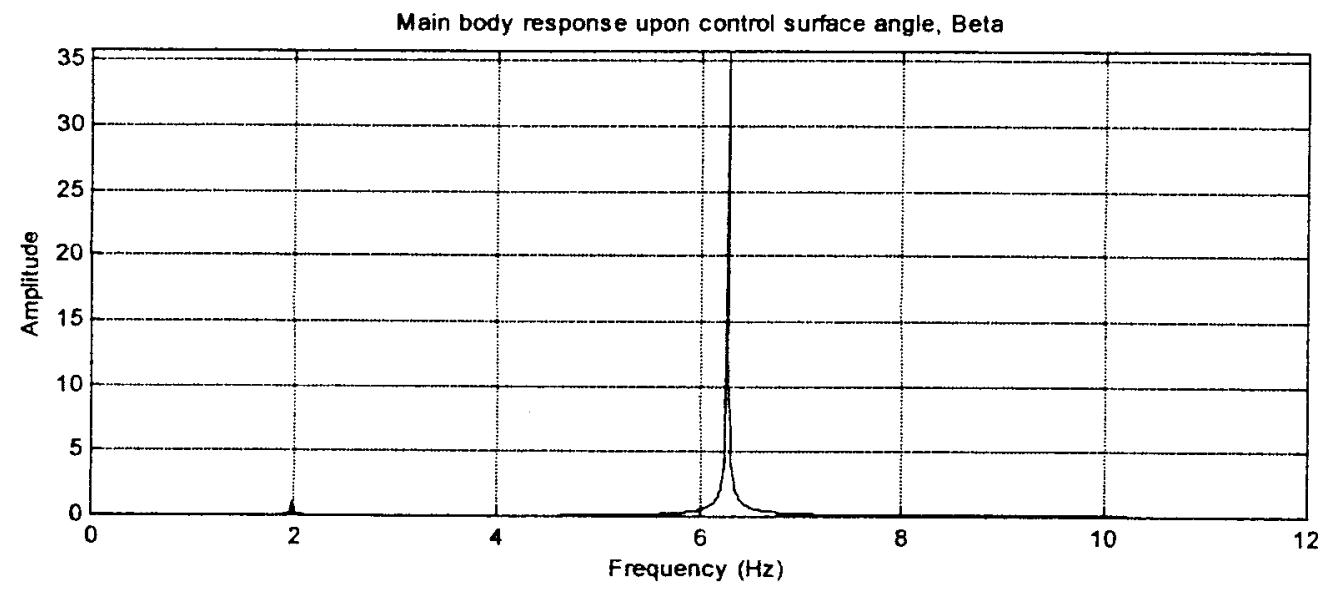

Fig. 13 Sample FRF between wing response and control angle at $20 \mathrm{~m} / \mathrm{s}$ velocity for the wing/control with actuator model

signal, when employing an actuator-type device to move the control surface, is the actual demand signal.

\section{EXPLANATION IN TRANSFER FUNCTION TERMS}

In order to attempt to explain further why the actuator force and control rotation angle are entirely inappropriate as reference signals for flutter testing, consider an argument based on transfer functions. It is well known that a transfer function comprises poles in the denominator and zeros in the numerator. The true poles of a system are unique whereas the zeros depend upon the actual input and response positions.

Now consider the response $Y(s)$ on the wing or control surface, where $s$ is the Laplace operator, expressed in terms of the input (or reference) signal demanded of the actuator $B(s)$. As this is the source of the excitation to the system, the transfer function between $Y(s)$ and $B_{i}(s)$ will contain the true poles of the system $P(s)$, so

$$
H_{Y B_{i}}(s)=\frac{Y(s)}{B_{i}(s)}=\frac{Z_{Y B_{i}}(s)}{P(s)}
$$

where $Z_{Y B_{i}}(s)$ contains the relevant zeros for this response/reference combination. Any test using this transfer function will inevitably yield the true stability characteristics of the flutter system, embedded in $P(s)$.

Now consider using the control rotation $B(s)$ (or actuator displacement) as the reference signal. Because this is actually part of the response of the system $\beta$ (just as much as $\gamma$ or $\theta$ ), it may be related to $B_{i}(s)$ as follows:

$$
B(s)=H_{B B_{i}}(s) B_{i}(s)=\frac{Z_{B B_{i}}(s)}{P(s)} B_{i}(s)
$$

Thus, using equations (30) and (31), the transfer function (or FRF) between the response $Y(s)$ and 


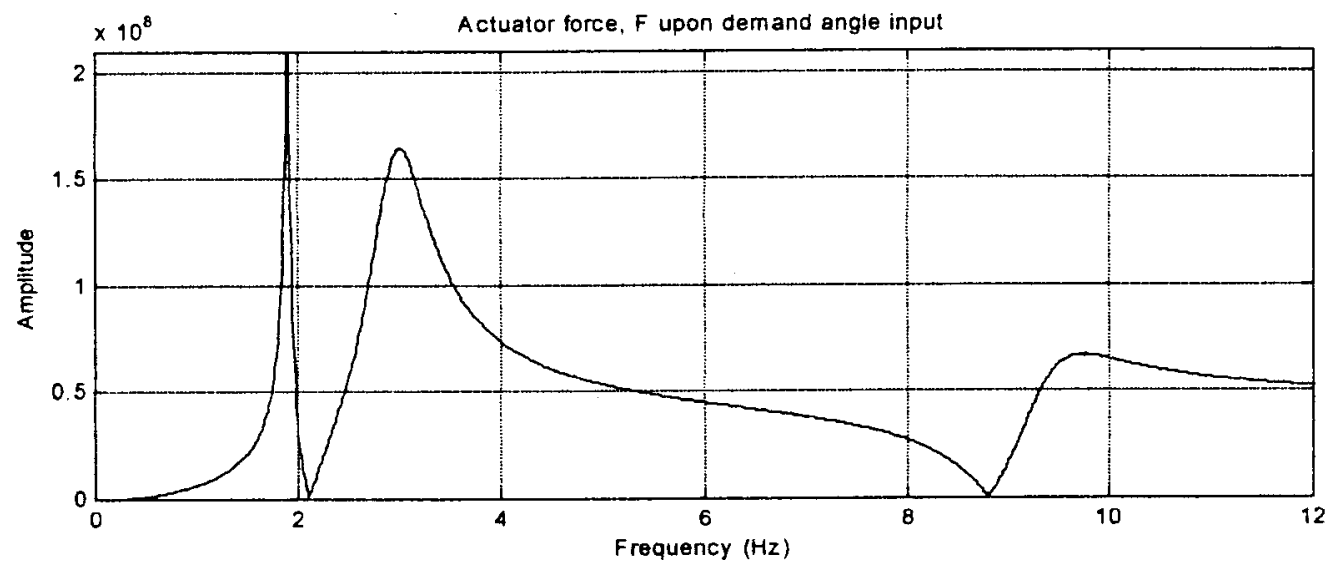

Fig. 14 Sample FRF between actuator force and demand angle at $20 \mathrm{~m} / \mathrm{s}$ velocity for the wing/control with actuator model

control rotation angle $B(s)$ may be written as

$$
H_{Y B}(s)=\frac{Y(s)}{B(s)}=\frac{\frac{Z_{Y B_{i}}(s)}{P(s)} B_{i}(s)}{\frac{Z_{B B_{i}}(s)}{P(s)} B_{i}(s)}=\frac{Z_{Y B_{i}}(s)}{Z_{B B_{i}}(s)}
$$

From this equation it may be seen that the apparent poles of this transfer function are actually the zeros of the control rotation/control demand transfer function and will therefore not contain the essential stability information in $P(s)$ for the flutter system. A similar argument applies to using the force in the actuator $F_{\mathrm{P}}(s)$
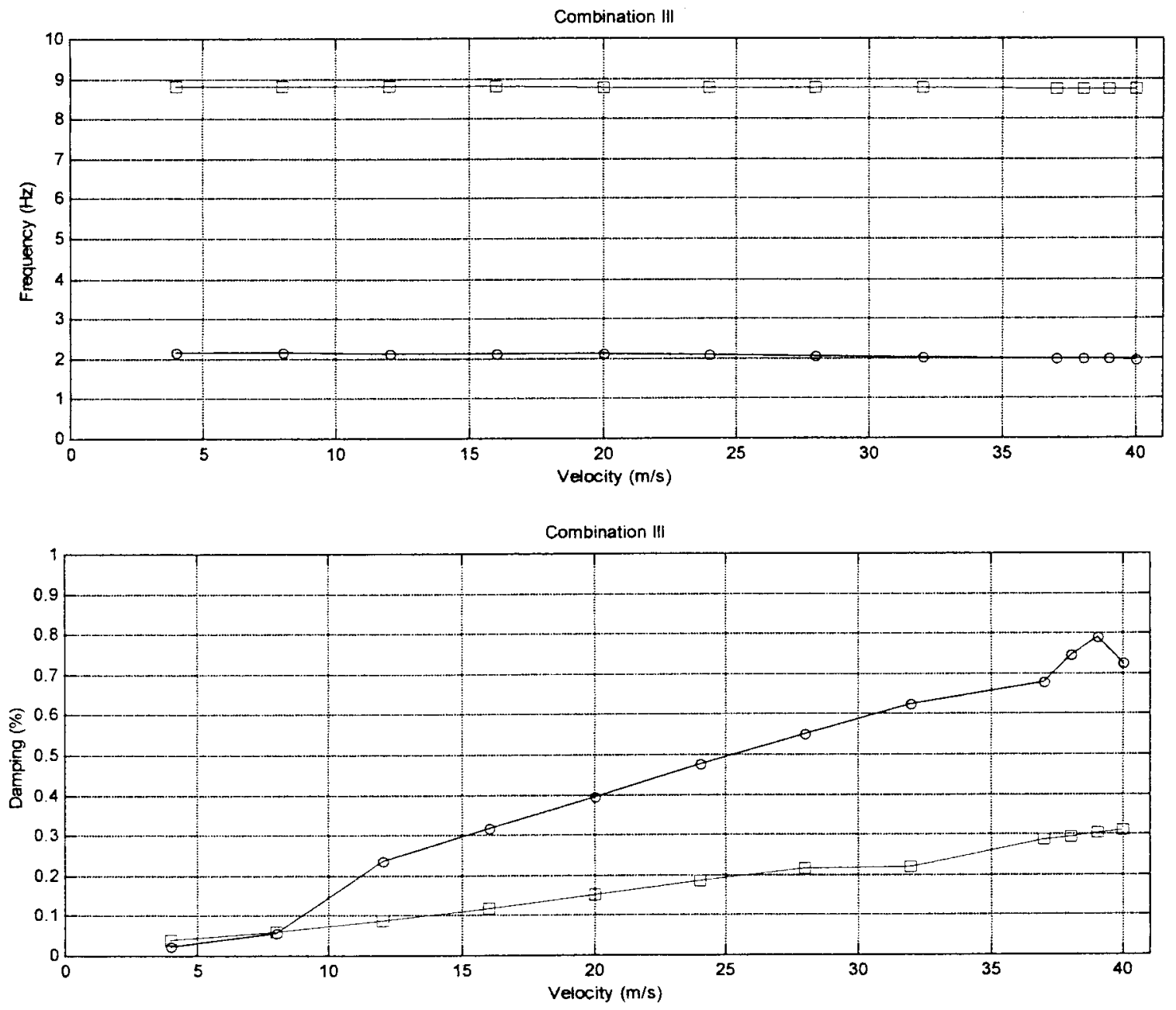

Fig. 15 Simulated flutter test results over the velocity range using actuator force as the reference for the wing/control with actuator model 

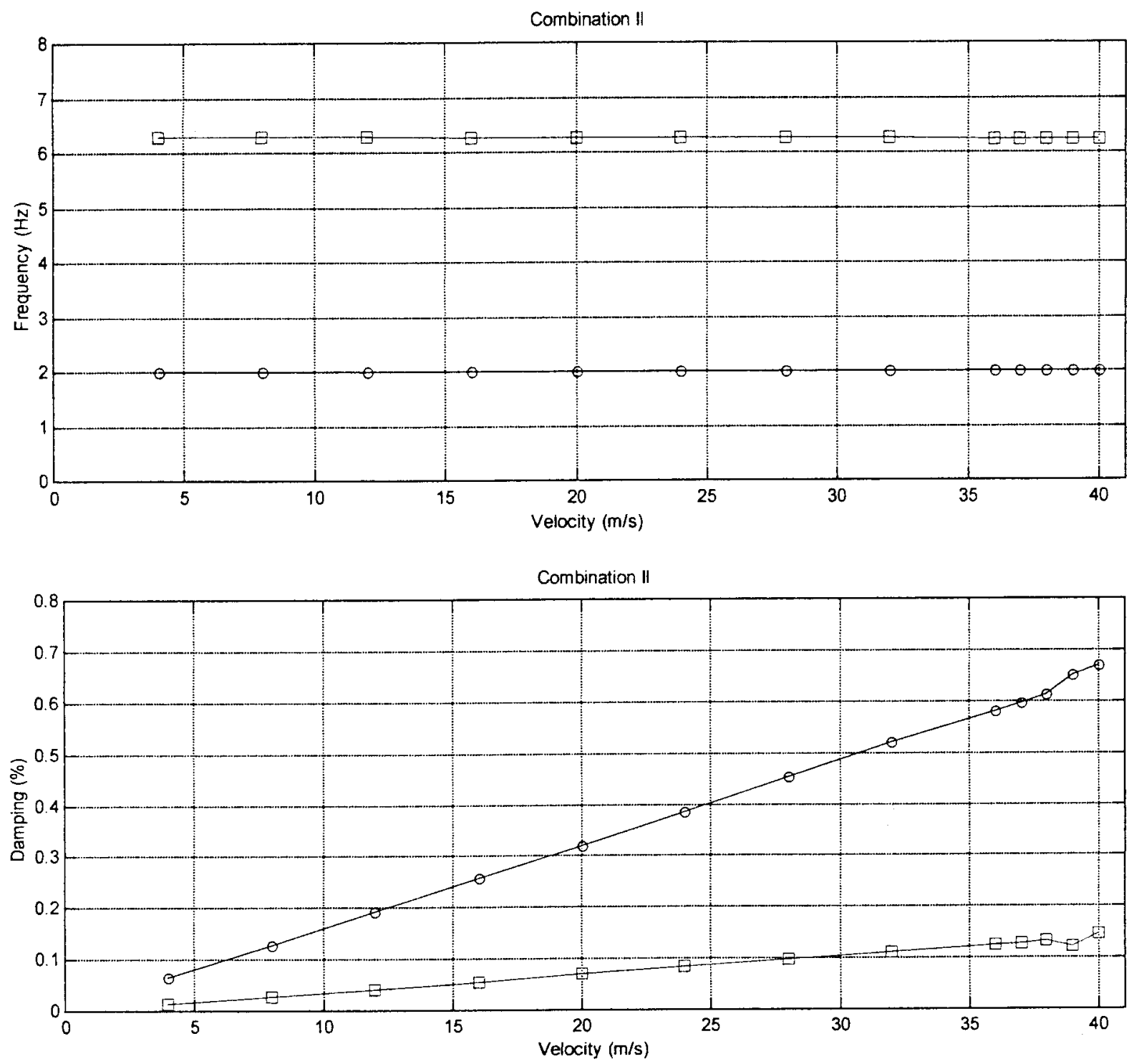

Fig. 16 Simulated flutter test results over the velocity range using control angle as the reference for the wing/control with actuator model

as a reference signal, but here the force is less directly linked to the response. The force derives from the pressure difference across the actuator chambers $P_{\mathrm{J}}$ (which is actually one of the states of the system) and thus the displacement of the actuator body, which is in turn directly linked to the control rotation by kinematics.

Thus the reason that the apparent test results were incorrect is that the characteristics of some of the system zeros were being followed instead of the poles. Because some of the zeros are close to the poles, some of the 'apparent' frequencies happened to be close to the true ones.

\section{IMPACT ON THE FLUTTER TEST PHILOSOPHY}

The lesson for flutter testing is very clear and simple. If an actuator is used to provide excitation via a control surface, then the actuator demand signal should be used as the reference signal for FRF calculation. To use the actuator force (or strain) or control rotation angle (or actuator body displacement) as a reference could yield incorrect and potentially misleading results and could even result in flutter occurring without warning.

In reference [5], it is recognized that the 'aileron 
rotational position' is not a true force signal, but nevertheless the resulting transfer function was analysed and results used for qualitative purposes only, to support sine dwell trends. In this case, because the aileron rotation angle turned out to be fairly constant with frequency, the results would have been satisfactory; however, had this not been the case, the availability of potentially incorrect data could have been misleading and could have given different trends.

It is interesting that in reference [6] results from analysis of a resonant dwell based on the acceleration response were compared to those from curve-fitting an FRF generated using an aileron motion sensor and a significant difference in frequency was found. Nonlinearity was blamed, but it could well be that the difference could also have been due to the reference signal chosen.

One check as to whether the use of, say, the control angle is likely to lead to incorrect results is whether the time history (in the case of a constant amplitude chirp) displays any variation in amplitude. If the amplitude is essentially constant then the control and actuator are not significantly involved in the flutter mechanism and test results will be satisfactory. For example, in reference [4], the time histories for the control deflections are fairly flat over the lower frequency range of interest and therefore the results shown are not likely to be incorrect.

\section{CONCLUSIONS}

In this paper, simulations of a 3 degree-of-freedom flutter system have been performed with control rotation restrained firstly by a spring and secondly by a simple hydraulic actuator. The actuator was used to provide excitation for a simulated flutter test and it was shown that the only appropriate reference signal for data processing was the control angle demand input to the actuator. Using the actuator force or measured control angle could lead to incorrect and potentially dangerous results in the flutter test.

\section{REFERENCES}

1 Scanlan, H. and Rosenbaum, R. Introduction to the Study of Aircraft Vibration and Flutter, 1951 (Macmillan, London).

2 Cooper, J. E. Parameter estimation methods for flight flutter testing. AGARD-CP-566, Advanced Aeroservoelastic Testing and Data Analysis, 1995.

3 van Nunen, J. W. G. and Piazzoli, G. Aeroelastic flight test techniques and instrumentation. AGARDograph 160, Vol. 9, Flight Test Instrumentation Series, 1979.

4 Bennett, R. M. and Abel, I. Application of a flight test and data analysis technique to flutter of a drone aircraft. In AIAA Dynamics Specialists Conference, Atlanta, Georgia 1981, AIAA paper 81-0652.
5 Dunn, S. A., Farrell, P. A., Budd, P. J., Arms, P. B., Hardie, C. A. and Rendo, C. J. F/A-18A flight flutter testing-limit cycle oscillation or flutter. In International Forum on Aeroelasticity and Structural Dynamics, Madrid, Spain, 2001.

6 Dickenson, M. CF-18 flight flutter test (FFT) techniques. AGARD-CP-566, Advanced Aeroservoelastic Testing and Data Analysis, 1995.

7 Hancock, G. J., Simpson, A. and Wright, J. R. On the teaching of the principles of wing flexure/torsion flutter. Aeronaut. J., 1985, 89(888), 285-305.

8 Richardson, M. and Formenti, D. L. Parameter estimation from frequency response measurements using rational fraction polynomials. In Proceedings of the 1st IMAC, 1982.

\section{APPENDIX 1}

\section{Wing/control model parameters}

The wing/control structure was assumed to have a uniform mass distribution of $\mu=1320 \mathrm{~kg} / \mathrm{m}^{2}$, span $s=$ $3.5 \mathrm{~m}$ and chord $c=0.7 \mathrm{~m}$. Also, $x_{\mathrm{f}}=0.4 c=0.28 \mathrm{~m}$ and $x_{\mathrm{h}}=0.75 c=0.525 \mathrm{~m}$ were chosen. The moments of inertia could then be calculated from first principles and had the following values: $I_{\gamma}=13205, I_{\theta}=148$, $I_{\beta}=8.25, I_{\gamma \theta}=396, I_{\gamma \beta}=123.8$ and $I_{\theta \beta}=25.6 \mathrm{~kg} \mathrm{~m}^{2}$.

Stiffness values were chosen such that the uncoupled natural frequencies (i.e. ignoring product moments of inertia coupling terms) for bending, torsion and control rotation were $2.0,6.0$ and $2.2 \mathrm{~Hz}$ to ensure that the flutter mechanism involved some control rotation. These natural frequency choices led to stiffness values of $k_{\gamma}=(2 \pi \times 2.0)^{2} I_{\gamma}=2.085 \times 10^{6}, \quad k_{\theta}=(2 \pi \times 6.0)^{2} I_{\theta}=$ $2.10 \times 10^{5}, \quad k_{\beta}=(2 \pi \times 2.2)^{2} I_{\beta}=1.576 \times 10^{3} \mathrm{~N} \mathrm{~m} / \mathrm{rad}$. Clearly, the frequencies for the coupled system are somewhat different to these assumed frequency values, namely $1.75,2.61$ and $9.11 \mathrm{~Hz}$. The aerodynamic parameters were estimated using equations found in Scanlan and Rosenbaum [1] and the following values were determined: $a=6.283, a_{\mathrm{c}}=3.826, a_{\mathrm{m}}=$ $-0.076, b_{1}=-0.035, b_{2}=-0.022, M_{\dot{\theta}}=-0.220$ and $M_{\dot{\beta}}=-0.035$.

\section{APPENDIX 2}

\section{Actuator characteristics}

Equation (22) in the paper can be simplified to

$$
d_{1} \dot{P}_{\mathrm{J}}+d_{3} P_{\mathrm{J}}=-A_{\mathrm{P}} \dot{X}_{0}-d_{2} X_{0}+d_{2} X_{i}
$$

where the parameters $d_{1}, d_{2}$ and $d_{3}$ are clear by direct comparison of equations (22) and (33). To examine the transmissibility and impedance characteristics of the basic actuator model, the differential pressure can be eliminated from equations (23) and (33). Using the 


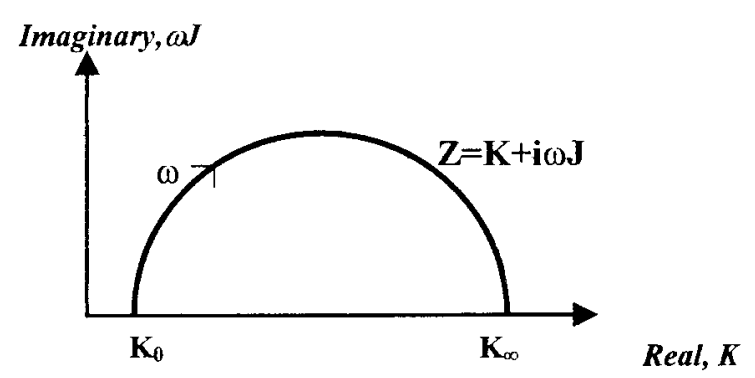

Fig. 17 Impedance of the actuator

Laplace $s$ domain,

$$
F_{\mathrm{P}}=A_{\mathrm{P}}\left(\frac{A_{\mathrm{P}} s+d_{2}}{d_{1} s+d_{3}}\right) X_{0}-\left(\frac{d_{2}}{d_{1} s+d_{3}}\right) X_{i}
$$

Setting $X_{i}=0$ (i.e. no demand), then equation (34) yields the actuator impedance $Z(s)$ :

$$
Z(s)=\frac{F_{\mathrm{P}}}{X_{0}}=A_{\mathrm{P}}\left(\frac{A_{\mathrm{P}} s+d_{2}}{d_{1} s+d_{3}}\right)
$$

Using $s=\mathrm{i} \omega$ and rationalizing the denominator of the complex equation yields the real and imaginary parts of the impedance:

$$
\begin{aligned}
Z(\omega) & =K+\mathrm{i} \omega J \\
& =A_{\mathrm{P}}\left(\frac{d_{2} d_{3}+\omega^{2} d_{1} A_{\mathrm{P}}}{d_{3}^{2}+\omega^{2} d_{1}^{2}}\right)+\mathrm{i} \omega\left(\frac{A_{\mathrm{P}} d_{3}-d_{1} d_{2}}{d_{3}^{2}+\omega^{2} d_{1}^{2}}\right)
\end{aligned}
$$

where $K$ and $\omega J$ are the stiffness and damping components respectively. The locus for $Z$ with variation in $\omega$ is the semicircle shown in Fig. 17. $K_{0}$ and $K_{\infty}$ are the static stiffness and oil bounce stiffness of the actuator respectively, namely

$$
K_{0}=\frac{A_{\mathrm{P}} d_{2}}{d_{3}}=\frac{\mu A_{\mathrm{P}} K_{\mathrm{F}}}{A_{\mathrm{F}}}, \quad K_{\infty}=\frac{A_{\mathrm{P}}^{2}}{d_{1}}=\frac{4 N A_{\mathrm{P}}^{2}}{d_{1}}
$$

In flutter calculations, the hydraulic actuator characteristics are not usually modelled in detail. Instead, values of $K$ are used for a spring to study the effect of a range of actuator stiffness values, whereas the damping contribution is often ignored, though it is by no means insignificant.

Putting $F_{\mathrm{P}}=0$ into the force expression yields the actuator displacement transmissibility $T_{\mathrm{D}}$ for zero load, giving

$$
T_{\mathrm{D}}(s)=\frac{X_{0}}{X_{i}}=\frac{d_{2}}{A_{\mathrm{P}} s+d_{2}}
$$

which is equivalent to a simple lag with cut-off frequency

$$
\omega_{\mathrm{D}}=\frac{d_{2}}{A_{\mathrm{P}}}=\frac{\mu K_{\mathrm{V}}}{A_{\mathrm{P}}} \sqrt{\frac{P_{\mathrm{S}}}{2}}
$$

In a similar way, putting $X_{0}=0$ yields the force transmissibility $T_{\mathrm{F}}$ for the 'blocked' actuator body, with a cut-off frequency of

$$
\omega_{\mathrm{F}}=\frac{d_{3}}{d_{1}}=\frac{4 K_{\mathrm{V}} A_{\mathrm{F}} N}{K_{\mathrm{F}} V} \sqrt{\frac{P_{\mathrm{S}}}{2}}
$$

\section{APPENDIX 3}

\section{Actuator model parameters}

The actuator parameters were selected to yield a stiffness value that gave a control rotation frequency similar to that for the basic spring, together with reasonable values of the cut-off frequencies. Also, because the transient solution of the equation in $P_{\mathrm{J}}$ includes the term $\exp \left(-d_{3} / d_{1}\right)$, care had to be taken with the decay rate compared to the time constant of the wing/control.

The actuator parameters were chosen as follows: $A_{\mathrm{P}}=7.068 \times 10^{-4} \mathrm{~m}^{2}, \quad V=5.301 \times 10^{-5} \mathrm{~m}^{3}, N=6.9 \times$ $10^{8} \mathrm{~N} / \mathrm{m}^{2}, P_{\mathrm{S}}=200000 \mathrm{~N} / \mathrm{m}^{2}, K_{\mathrm{V}}=0.003, \mu=0.05$, $A_{\mathrm{F}}=7.854 \times 10^{-7} \mathrm{~m}^{2}, \quad K_{\mathrm{F}}=34000 \mathrm{~N} / \mathrm{m} \quad$ and $h=$ $0.04 \mathrm{~m}$. Thus $K_{0}=1.53 \times 10^{6} \mathrm{~N} / \mathrm{m}, \quad K_{\infty}=2.6 \times$ $10^{7} \mathrm{~N} / \mathrm{m}, f_{\mathrm{D}}=10.7 \mathrm{~Hz}$ and $f_{\mathrm{F}}=181 \mathrm{~Hz}$. The natural frequencies for the coupled wing/control plus actuator system at zero velocity are $1.87,3.00$ and $9.33 \mathrm{~Hz}$ compared to the values of $1.75,2.61$ and $9.11 \mathrm{~Hz}$ for the basic system with a simple spring. Note that the actual values of the parameters are not critical in demonstrating the issues in this paper. 
Copyright of Proceedings of the Institution of Mechanical Engineers -- Part G -Journal of Aerospace Engineering is the property of Professional Engineering Publishing and its content may not be copied or emailed to multiple sites or posted to a listserv without the copyright holder's express written permission. However, users may print, download, or email articles for individual use. 